\title{
NeuroImage
}

\section{Specialization within the ventral stream: the case for the visual word form area}

\author{
Laurent Cohen $^{\mathrm{a}, \mathrm{b}, *}$ and Stanislas Dehaene ${ }^{\mathrm{b}}$ \\ ${ }^{a}$ Institut de Neurologie, Hôpital de la Salpêtrière, AP-HP, Paris, France \\ ${ }^{\mathrm{b}}$ INSERM U562, Service Hospitalier Frédéric Joliot, CEA/DSV, Orsay, France
}

Received 13 September 2003; revised 11 December 2003; accepted 23 December 2003

Is there specialization for visual word recognition within the visual ventral stream of literate human adults? We review the evidence for a specialized "visual word form area" and critically examine some of the arguments recently placed against this hypothesis. Three distinct forms of specialization must be distinguished: functional specialization, reproducible localization, and regional selectivity. Examination of the literature with this theoretical division in mind indicates that reading activates a precise subpart of the left ventral occipitotemporal sulcus, and that patients with pure alexia consistently exhibit lesions of this region (reproducible localization). Second, this region implements processes adequate for reading in a specific script, such as invariance across upper- and lower-case letters, and its lesion results in the selective loss of reading-specific processes (functional specialization). Third, the issue of regional selectivity, namely, the existence of putative cortical patches dedicated to letter and word recognition, cannot be resolved by positron emission tomography or lesion data, but requires high-resolution neuroimaging techniques. The available evidence from single-subject fMRI and intracranial recordings suggests that some cortical sites respond preferentially to letter strings than to other categories of visual stimuli such as faces or objects, though the preference is often relative rather than absolute. We conclude that learning to read results in the progressive development of an inferotemporal region increasingly responsive to visual words, which is aptly named the visual word form area (VWFA).

(c) 2004 Elsevier Inc. All rights reserved.

Keywords: Word recognition; Occipitotemporal; Specialization

\section{Introduction}

The efficiency of reading in literate adults rests on the ability to quickly identify visual words across large variations of irrelevant parameters such as position, size, color, font, or case. This perceptual expertise requires no less than 5 years of academic training in a specific writing system (Aghababian and Nazir, 2000). The outcome of this perceptual normalization process is an abstract representation of letter identities that has been termed the visual

* Corresponding author. Service de Neurologie 1, Hôpital de la Salpêtrière, 47/83 Bd de l'Hôpital, Paris CEDEX 13, 75651, France. Fax: +33-1-44-24-52-47.

E-mail address: laurent.cohen@psl.ap-hop-paris.fr (L. Cohen).

Available online on ScienceDirect (www.sciencedirect.com.) word form (Riesenhuber and Poggio, 1999; Warrington and Shallice, 1980). We formulated the idea that an area in the midportion of the left fusiform gyrus, which activates whenever literate subjects are presented with strings of letters, contributes crucially to the cerebral basis of the visual word form. Accordingly, we proposed to label this left fusiform region as the visual word form area (VWFA) (Cohen et al., 2000). This hypothesis was based both on neuroimaging studies of reading and on anatomo-clinical correlations in patients with pure alexia, an acquired deficit of reading that follows left occipitotemporal lesions (McCandliss et al., 2003). The VWFA hypothesis was framed in the broader context of recent studies of functional specialization within the human ventral visual stream, in which have been identified several areas specialized for other classes of visual stimuli such as faces, places, or body parts (Downing et al., 2001; Hasson et al., 2003; Haxby et al., 2001; Kanwisher et al., 1997).

In a recent article, Price and Devlin (2003, hereafter P\&D; see also Price et al., 2003) challenge this view by a critical examination of neuropsychological and neuroimaging evidence. In a nutshell, they claim that imaging studies show activations of the VWFA in a broad set of experimental conditions, including reading, but also auditory words processing, object perception, naming, perception of "socially interactive" movements, and so on. P\&D suggest that, whatever the actual function(s) of this region, its activation in such a diversity of conditions runs against the idea of any specialization for alphabetic processing. Similarly, they assert that the extant neuropsychological evidence lends no support to the VWFA hypothesis. They claim, first, that pure alexia cannot be ascribed to lesions of the VWFA, and, second, that it results from a general visual impairment rather than from a specific reading deficit.

In the present article, we try to bring the debate on the VWFA back to fundamental issues of cortical specialization and their testability with neuroimaging and neuropsychological methods. We first consider the basic computational challenges that the visual system of skilled readers must meet to process letters and words with high efficiency. We show that it must achieve perceptual invariance, parallel letter processing, and tuning to a specific writing system. We argue that such perceptual expertise is likely to require specialized neural systems within the ventral visual stream.

What does "specialized" mean, however? We suggest that the muddled concept of specialization should be broken down into three independent issues: functional specialization, reproducible 
cortical localization, and regional selectivity. This allows us to articulate detailed hypotheses and predictions relative to the VWFA. We then review relevant evidence from functional neuroimaging, with an emphasis on methods with a high spatial resolution, which are most critical to the debate. We turn next to neuropsychological studies of patients with pure alexia, which reveal the consequences of a disrupted visual word form system following left occipitotemporal lesions. We show that both neuroimaging and neuropsychological studies lend convergent support to the hypothesis of the VWFA.

\section{Theoretical issues in visual word recognition}

\section{The necessity of specialization for visual word recognition}

We start with a computational analysis of the visual word recognition process. What type of problems does the visual system of a literate person routinely solve? A first problem is invariance. We are able to recognize words across considerable changes in font, CASE, location, and size. Even highly unfamiliar word forms, such as mIxEd CaSe, do not pose much difficulty for our visual system (Besner, 1989; Mayall et al., 1997; Paap et al., 1984). This implies that the visual word recognition process is highly efficient in "normalizing" and computing an invariant representation that discards irrelevant variations in the visual input. At the same time, however, very small details that are relevant to reading are maintained and even amplified. Consider the words "eight" and "sight". We are immediately aware of their radically different meanings and pronunciation, but it takes some time to realize that visually speaking, those words differ only by a very small amount. Our visual system is attuned to the minute difference between "eight" and "sight", which it amplifies so that we ultimately address completely different regions of lexical space, although it discards the much greater differences between "eight" and "EIGHT". Those computational requirements are largely common to the recognition of any type of visual objects: Tools, buildings, or faces can all appear with huge variations in their appearance, including size, location, orientation, and so on. Like with words, the visual system must both compensate for irrelevant variations in the input and exploit specific details that are critical for object identification (Riesenhuber and Poggio, 1999).

In the case of reading, crucial aspects of this capacity of invariant recognition must be learned. A general process of size invariance may suffice to recognize the identity of, say the letters "o" and "O". In general, however, what constitutes a significant visual difference and what does not is defined culturally rather than visually. Many of the mappings between upper case and lower case, for instance, are arbitrary. The shape "e" might have been selected as the lower case of " $\mathrm{A}$ "- it is only a historical accident if the shape " $a$ " was chosen. Thus, the fast mapping of "A" and "a" onto the same abstract letter identity cannot be explained by generic features of the organization of the visual system. Rather, the case invariance observed in several psychological experiments (e.g. Besner et al., 1984; Bowers et al., 1998) must arise from specialization resulting from a learning process. Indeed, readers of the Latin script, who immediately perceive a difference between the letters e and c, may not know that the Hebrew letters $\Pi$ and $\Pi$ are distinct, something that will be obvious to any reader of that script.
Beyond case invariance, other psychological findings provide evidence for a functional tuning in reading. The word superiority effect is the fact that when asked to detect the presence of a letter, subjects typically show superior performance when the letter is embedded in a visual string that respects the structure of the learned language than when it is embedded in a random string. For instance, subjects are faster and more accurate in detecting the letter "w" in "show" than in "ohsw" (Reicher, 1969). This phenomenon, which is found with real words, pseudo-words (Spoehr and Smith, 1975), and even nonwords sufficiently similar to words such as SPCT (Rumelhart and McClelland, 1982), implies the existence of a mechanism tuned to the possible structures of visual words. Also remarkable is the absence of any effect of word length: within a range of about three to six letters, visual word recognition takes a constant time regardless of the number of letters composing that word (Lavidor and Ellis, 2002; Weekes, 1997). This suggests the presence of a parallel apprehension mechanism specialized for reading. Note that the absence of a length effect is found only when the words are presented in the familiar horizontal format and within a restricted region of the fovea and right hemifield (Lavidor and Ellis, 2002; Lavidor et al., 2001). This suggests that at least part of the specialization for letter and word recognition occurs at retinotopically organized stages of visual processing (Nazir, 2000).

All in all, both computational arguments and experimental psychological data point to specialization for visual word recognition in skilled readers. Contrary to what is stated by P\&D, it is not just that "reading is more complex and imposes greater demands on visual processing" than identifying pictures, faces, or colors. Rather, reading poses particular problems to the visual system, some of which (such as case invariance) are specific to reading in a given script. A generic visual recognition device is simply insufficient to account for the notation-dependent operations that are observed in skilled readers.

\section{Three forms of specialization}

How, then, is invariant visual word recognition implemented in neural tissue? When considering the mapping from psychological models to their cortical implementation, we argue that three distinct theoretical possibilities must be carefully distinguished: functional specialization, reproducible localization, and regional selectivity. These can be considered as increasingly local forms of cortical specialization.

Functional specialization refers to the possibility that the visual system has become, at least in part, attuned to the requirements of reading in a given script. For instance, if some visual neurons fired identically to "A" and "a", but not to other letters, this would indicate abstract case invariance and would constitute evidence of functional specialization for reading in Latin script. Likewise, if some neurons responded to various forms of the letter $\boldsymbol{\Pi}$, but did not respond to $\Pi$, this would constitute functional specialization for reading in Hebrew. Note, however, that the hypothesis of functional specialization need not be associated with any hypothesis about the localization of such processes. It is logically possible that case-invariant neurons are present throughout the visual system, without any particular localization.

An alternative hypothesis is that such neurons tend to be grouped together in some fixed regions of visual cortex. We call this the reproducible localization hypothesis. Importantly, this hypothesis only claims that neurons engaged in the reading process 
are not localized randomly; it makes no claim about the localization of neurons engaged in other processes such as face, object, or color processing. The same neurons could be involved, or a different subset of neurons within the same region, or an entirely different patch of cortex.

Yet, a third logically independent hypothesis is what we call the regional selectivity hypothesis. This is the postulate that there are regions of cortex devoted solely to word reading. According to the regional selectivity hypothesis, at a suitably small scale (e.g. 1 or 2 $\mathrm{mm}$ ), it should be possible to identify patches of cortex that respond exclusively to letters or words, and do not respond at all to stimuli such as faces or objects that do not contain features of letters or words.

\section{The hypothesis of the visual word form area}

With those distinctions in mind, we can now state our position quite clearly. We propose that there is functional specialization for reading in the brain of literate human subjects. We also propose that there is reproducible localization of the neural circuits that are attuned to the reading process. In particularly, within the visual system, we claim that whenever subjects read a word, a reproducible portion of the left occipitotemporal sulcus is activated and hosts functionally specialized circuits for letter and word recognition. We further claim that this area can be reproducibly observed in all cultures, even in readers of nonalphabetic scripts.

We suggest that this reproducible localization constitutes sufficient grounds for christening this region the visual word form area. We do not, however, believe that there is complete regional selectivity for word recognition. Even the voxels that respond optimally to words tend to be also activated by other stimuli such as pictures or line drawings of objects. This should not be surprising because the functional specialization for reading arises from a partial preemption or "recycling" of visual cortex which evolved for other purposes during phylogenesis (Dehaene, 2004). This cortex is partially plastic and can, during ontogenesis, become progressively attuned to the requirements of reading (McCandliss et al., 2003). In the most expert readers, one might perhaps identify patches of cortex that respond to written words more than to any other visual stimuli. In most subjects, however, the specialization for words is likely to be only partial. There may be columns of neurons specialized for the recognition of letters, graphemes, or words, but they are likely to be intermixed with other neurons involved in object and face recognition (Puce et al., 1999). Therefore, at the scale accessible to human neuroimaging, most if not all of the cortical surface is likely to respond jointly to words, faces, and objects, with smooth but systematic changes in local selectivity (Haxby et al., 2001).

Importantly, such overlap between activations to words, objects, and faces does not preclude studying the functional contribution of inferior temporal cortex to reading per se. The issue of the nature of the contribution of a given cortical sector to reading (e.g. Is it caseindependent? Is it location-invariant? Does it code for single letters, or for graphemes, syllables, or morphemes?) can be addressed completely independently of the orthogonal issue of whether neurons in this cortical sector also contribute to object or face recognition.

The term VWFA was introduced to refer to the simple fact that a reproducible cortical region is active during visual word recognition and appears to possess properties of location and case invariance that suggest functional specialization for reading (Cohen et al., 2000, 2002; Dehaene et al., 2001). Our use of the word "area" may have caused some confusion. "Area" is used here solely as a synonym for "location". It is not meant in the more technical sense of a distinct cortical sector distinguished by its layer, cellular, or receptor structure as used for instance in the term "Brodmann area". We do not believe that there is a cytoarchitectonically defined subpart of the visual system that responds to visual words. We also consider it rather unlikely that the visual word area would have sharp borders that would delineate it qualitatively from other neighboring areas involved in face or object recognition. Rather, the density of neurons activated during reading probably varies continuously, though nonrandomly, across the surface of the ventral occipitotemporal cortex, thus creating a landscape of local preference for various categories of objects (Hasson et al., 2002; Haxby et al., 2001). Finally, it is likely that the rather large occipitotemporal region that we have termed VWFA can be further subdivided into, for instance, a posterior location-specific sector and a more anterior location-invariant sector (see Dehaene et al., in press). None of this, however, detracts from the usefulness of having a single term to refer to the anatomically reproducible ventral visual region that activates focally during word reading.

\section{Evidence from functional neuroimaging}

\section{Methodological issues}

The above discussion suggests two possible research directions to study the visual word form hypothesis using neuroimaging methods. The first direction focuses on probing the reproducible localization and regional selectivity of brain activations during visual word recognition. This requires neuroimaging techniques with the highest possible spatial resolution, such as fMRI, MEG, or intracranial recordings, to examine whether some (presumably small) patches of cortex are partially or entirely dedicated to word recognition, and whether those are reproducibly observed at a roughly identical location in all subjects. The second direction of research consists in probing the functional specialization of the ventral fusiform region to reading-specific processes, such as case invariance. Because fine spatial localization is not critical, this second strategy can potentially be studied with coarser brainimaging methods such as PET or ERPs. However, it requires using carefully designed minimal contrasts adequate to address issues of functional organization.

Unfortunately, in their critique of the visual word form area, P\&D adopt neither of those stances. Their argument relies almost exclusively on the overlap of data from different groups of subjects scanned with PET. However, such data are typically generated with a spatial smoothness of $15 \mathrm{~mm}$ in PET group studies. In this context, the finding of overlap between wordrelated and non-word-related (e.g. color or object processing) tasks is not conclusive, as it seems likely that even cortical territories known to be distinct would be found to overlap with this method. This is especially true when the method is applied across different groups of subjects, although individual sulci are known to vary at least up to $1 \mathrm{~cm}$ (Sowell et al., 2002; Thompson et al., 1996).

Many research groups are struggling to increase the spatial resolution of human data, for instance, by using high-field fMRI rather than PET, by repeatedly scanning the same subjects and 
reporting single-subject data, and by comparing fMRI results with MEG or intracranial recordings. Several studies, none of which are cited by $P \& D$, have applied these approaches to the issue of cortical specialization for word recognition (Allison et al., 1994, 1999; Gauthier et al., 2000; Hasson et al., 2002, 2003; Nobre et al., 1994; Puce et al., 1996; Tarkiainen et al., 1999).

PET data remain appropriate to study functional rather anatomical specialization for word processing. Indeed, some of the first evidence for a specific response to structured letter strings, as opposed to matched visual stimuli, came from PET (Petersen and Fiez, 1993; Petersen et al., 1990). However, what is needed then is an experimental design with functionally meaningful contrasts. Merely examining the large-scale circuits involved in picture naming or in thinking about the meaning of heard words, and finding that part of this circuit overlaps with the coordinates of the visual word form area, as done by $\mathrm{P} \& \mathrm{D}$, is not sufficient to infer the functional contribution of this area. It is possible that the overlap is merely due to the coexistence of distinct, functionally unrelated circuits within approximately the same region. Only advanced experimental designs, such as the priming or adaptation method (Grill-Spector and Malach, 2001; Naccache and Dehaene, 2001), are apt at proving the presence of shared circuitry. P\&D fail to discuss the detailed evidence that has been obtained from such paradigms, although it provides evidence for functional specialization for case-invariant letter recognition in the left fusiform region (Dehaene et al., 2001; Dehaene et al., in press).

In what follows, we attempt to fill the gaps in P\&D's review of the neuroimaging evidence. First, we examine the evidence for localization and regional selectivity for letters and words in the left fusiform region, mostly from high-resolution single-subject fMRI and intracranial recordings. Second, we examine the evidence for functional specialization for reading-specific processes.

\section{Evidence for reproducible localization}

A large number of studies, using various methods, have evidenced a reproducible activation of the left fusiform gyrus during reading. Activation in this region can be identified merely by contrasting word reading relative to rest, either with PET or with fMRI (Beauregard et al., 1997; Brunswick et al., 1999; Dehaene et al., 2002; Fiez et al., 1999; Paulesu et al., 2000; Wagner et al., 1998). This region appears as a likely source of electrical and magnetic fields that are recorded over the left ventral occipitotemporal region, with a latency of about 150-200 ms, whenever subjects see words (Allison et al., 1994, 1999; Cohen et al., 2000; Nobre et al., 1994; Salmelin et al., 1996; Simos et al., 2002; Tarkiainen et al., 1999). When word reading is contrasted to a resting period, the active regions obviously comprise a large unspecific component of visual activity. However, simple subtractions allow a more specific delineation of the VWFA. For instance, Price et al. (1996) have obtained stronger activations to strings of consonants than to strings of pseudo-letters at coordinates close to the VWFA. In one study, we isolated the VWFA using a spatial invariance criterion: contrary to more posterior retinotopic areas, the VWFA is activated by words presented in either the left or the right hemifield (Cohen et al., 2000). In another, we further required this region to respond more to strings of letters than to chequerboards presented in either hemifield (Cohen et al., 2002). Other investigators have subtracted the activations induced by foveal words and by pictures of faces, textures, or buildings (Gauthier et al., 2000; Hasson et al., 2002; Puce et al., 1996). There is good convergence to the same approximate Talairach coordinates across those studies (Cohen et al., 2000).

Several fMRI papers have further shown that this area can be identified in single subjects (Cohen et al., 2000, 2002; Dehaene et al., 2002; Gauthier et al., 2000; Puce et al., 1996). This allows quantification of the interindividual variability, which is remarkably low. The VWFA is found at the same location in Talairach space (approximately $-43,-54,-12$ ) with a standard deviation of only about half a centimeter. ${ }^{1}$ Essentially all subjects show wordinduced activation in the left hemisphere, whereas a symmetrical right-sided activation is only found in a few subjects and at a weaker level. Similar results have been obtained using singlesubjects MEG (Tarkiainen et al., 1999) and intracranial recordings (Allison et al., 1999).

\section{Evidence for partial regional selectivity}

P\&D do not deny the reproducibility of fusiform activations during reading, but claim that the same voxels are activated in a broad variety of PET studies. However, they fail to cite several higher-resolution studies that report a partial selectivity for visual words within the ventral fusiform region. As noted above, we should perhaps not expect to find entire voxels solely responsive to visual words and not to any other category of stimuli. Nevertheless, the available evidence reveals systematic and reproducible activations that are greater to visual words than to other control stimuli.

Puce et al. (1996) report single-subject activations during passive presentation of visual words, faces, and textures. Nine out of twelve subjects showed greater activation to words than to faces in the left occipitotemporal sulcus, although a neighboring but slightly more mesial site conversely showed preferential activation to faces than to words. This systematic topographical organization was reproduced by Gauthier et al. (2000). Hasson et al. (2002) also used fMRI to contrast activations to words, houses, and faces. Again, a site with greater responsivity to words than to either faces or buildings was found in the left occipitotemporal sulcus, lateral to face- and building-related activations. This region showed the same selectivity whether the stimuli were presented foveally or peripherally, confirming a previous demonstration of location invariance in this region (Cohen et al., 2000). Hasson et al. (2003) suggest that the visual word-related activation always falls at a systematic location at the boundary between object-selective and face-selective regions in a large-scale map of object preference. A similar though slightly more complex picture emerges from intracranial recordings in epileptic patients (Allison et al., 1999; Nobre et al., 1994; Puce et al., 1999). Using subdural electrode arrays, Allison et al. $(1994 ; 1999)$ observed sites that showed fast P150 or N200 waveforms that were larger, or occasionally exclusive for letter strings compared to a variety of control stimuli such as phase-scrambled strings, flowers, faces, or geometrical shapes. The anatomical distribution of those sites also concentrates on the left occipitotemporal region, though with a greater spatial dispersion than suggested by comparable fMRI

\footnotetext{
1 Note that this applies only to the peak coordinates: the activation typically occupies an extended antero-posterior strip of cortex, which may span some subregions with partially distinct functions in the cortical hierarchy (Dehaene et al., in press; see below). Furthermore, the standard deviation of about $5 \mathrm{~mm}$ is true across multiple subjects within a given fMRI study. Across studies, the variability can be somewhat higher, most likely due to differences in methods used for normalizing brain anatomies.
} 
studies. On the basis of these data, Puce et al. (1999) suggest a theoretical model of spatially segregated cortical columns, each selective to a different type of visual stimuli, organized in local patches a few millimeters aside. Single-neuron studies (e.g. Kreiman et al., 2000) will be needed to assess whether there is absolute selectivity for words in some patches, or whether there is partial intermixing at the microscopic level, as suggested by optical imaging studies in monkeys (e.g. Wang et al., 1996). However, in the present state of knowledge, it cannot be denied that small subregions of visual cortex show at least partial selectivity for letter strings.

P\&D and Price et al. (2003) raise the issue of whether the VWFA is strictly involved in visual word processing. In Cohen et al. (2002), we indeed listed several studies that reported ventral fusiform activation during spoken word processing. However, do these activations overlap with the VWFA? Cohen et al. (2002) reviewed existing studies of ventral temporal activations by visual and nonvisual words. As already noted by Büchel et al. (1998), words in modalities other than the visual yielded activations more anterior (average $y=-43$ ) than those typical of the visual word form (average $y=-60$ ). Furthermore, the anterior activations were sensitive to the semantic demands of the task, whereas the posterior activations were observed even for visual pseudo-words relative to random letter strings. Thus, we suggest that the visual word form area must be carefully distinguished from more anterior regions that are increasingly cross-modal and engaged in semantic computations. Such a distinction is supported by several neuroimaging studies (e.g. Booth et al., 2002b; see review in Giraud and Price, 2001) as well as by intracranial recordings (Nobre et al., 1994, 1998).

In a recent fMRI study, we contrasted the processing of written and spoken words within single-subjects during a same-different task (Dehaene et al., 2002). In agreement with our hypotheses, in every subject, the left posterior fusiform gyrus was activated by visual words but there was no trace of activation by spoken words. This indicates clearly that this region is not a supramodal area and is not automatically activated during auditory word processing.

This does not, however, preclude the possibility of its top-down activation by auditory words. Thus, Booth et al. (2002a, 2003) observed an activation of the VWFA (TC -45, -57, -12) when subjects engaged in a spelling task on auditory words, but not when they engaged in a rhyming task on the same stimuli. Those results suggest that posterior inferotemporal activations during auditory word processing are optional and contingent on the engagement of the subject in orthographic processing. ${ }^{2}$

More work will be needed to understand which experimental conditions cause such top-down orthographic recruitment. For instance, Price et al. (2003) observed VWFA activation with PET during the repetition of spoken words, relative to a baseline condition of saying "OK" to auditory noises. Is it implausible, however, to suggest that spoken word repetition, especially in the context of a block-design PET experiment, causes a small top-

\footnotetext{
2 Burton et al. (2000) observed a left inferotemporal activation (TC $-58,-56,-8)$ when subjects performed same-different judgments on spoken syllables, but only when they had to extract the first phoneme of the syllable, not when they could base their decisions on the syllable as a whole. This region, which is ascribed to the inferior temporal gyrus, seems to be more lateral than the VWFA. It may rather overlap with a distinct inferior lateral cross-modal region with strong effects of task structure (Cohen et al., in preparation).
}

down activation of orthographic representations? We think that this is not so unlikely. It is known that the acquisition of literacy modifies the brain network involved in speech repetition (especially for pseudo-words, see Castro-Caldas et al., 1998). Furthemore, a dramatic interference of orthography on speech perception has been evidenced in a variety of behavioral tasks including rhyme detection (Donnenwerth-Nolan et al., 1981), syllable monitoring (Taft and Hambly, 1985), lexical decision (Ziegler and Ferrand, 1998), and phoneme monitoring. For instance, the interference of orthography prevents the detection of the phoneme " $p$ " in the spoken French word "absurde" (Hallé et al., 2000).

PET and FMRI studies are ill-adapted to resolve the issue of top-down or bottom-up posterior inferotemporal activations because they cannot resolve the fine temporal dynamics of neural activity. However, recent work using MEG to compare spoken and written word processing confirms that posterior inferior temporal activation is present early on (approximately $170 \mathrm{~ms}$ ) when reading words, but are absent or confined to the late part of the epoch when hearing words (Marinkovic et al., 2003, Figs. 1 and 4).

When considering bottom-up and top-down activations of the VWFA, it is also important to keep in mind the spatial limitations of imaging methods. High-resolution single-subject studies are needed to evaluate the claim that the very same region is activated in both cases. Recently, we performed a study analogous to Booth et al. (2002a, 2003) using an orthogonal design with factors of modality (visual or auditory words) and task (phoneme or grapheme detection) (Cohen et al., in preparation). Consistent with a topdown effect, we observed a left posterior inferotemporal activation even when spoken words were presented, and with a greater intensity during the grapheme task than during the phoneme task. In a group analysis, this activation overlapped with the VWFA, as defined by a left inferior temporal activation that was greater to visual than to spoken words. However, in single subjects, the two regions appeared as distinct but very close. The left occipitotemporal sulcus (average coordinates $-44,-68,-4$ ) was activated only by visual words, not by spoken words. Another more lateral and slightly more anterior inferotemporal region $(-48,-60,-16)$ was activated also by spoken words, with modulation by the graphemic task. This study points to a pitfall of the method favored by $P \& D$, that is, the intersection of a series of individual contrasts: this method is inherently limited by image resolution and can lead to inappropriate generalizations about the function of a given patch of cortex. It is likely that, as our ability to image this region evolves, more subdivisions will appear within what we currently think of as a single VWFA. Recently, for instance, we showed that its posterior part contains case-invariant but not location-invariant letter detectors, whereas its middle part is invariant across both case and location and may be sensitive to units larger that a single letter (see Dehaene et al., in press).

\section{Functional specialization}

Aside from the issue of regional selectivity, neuroimaging methods have also been used to address the issue of functional specialization for visual word recognition. To demonstrate functional specialization in the VWFA, one should exhibit a pattern of activation that cannot be reduced to generic visual processes, but necessarily reflects regularities of the writing system. Evidence of this type has been presented by several investigators.

First, there are indications that the VWFA is tuned to the shape of letters, relative to visually equivalent pseudo-letters. Following 
up on the initial insight of Petersen et al. (1990), Price et al. (1996) observed stronger activation in the left fusiform gyrus to strings of consonants than to strings of false-font characters. There is also evidence that strings of letters cause greater left fusiform activation than visually equivalent strings of digits (Polk et al., 2002). Indeed, intracranial recording suggests that there may be specialized subregions for letters vs. digits in the ventral occipitotemporal cortex (Allison et al., 1994). Such a fine-grained specialization cannot be explained by a generic visual process.

Second, the VWFA responds more to words or to pseudo-words than to random consonant strings that violate orthographic constraints (Beauregard et al., 1997; Büchel et al., 1998; Cohen et al., 2002; Price et al., 1996; Rees et al., 1999; Xu et al., 2001). ${ }^{3}$ This suggests that it has become sensitive to orthographic rules in the subject's language, which are matters of cultural convention.

Third, there is also evidence that the VWFA computes the arbitrary mapping between upper- and lower-case letters. The VWFA shows equally robust fMRI responses when words are presented in a familiar format or in a mixed-case format (e.g. "tAbLe") (Polk and Farah, 2002). Furthermore, the VWFA shows repetition priming whenever a target word is preceded by a subliminal presentation of the same word, whether the two words are printed in the same or in a different case (e.g. table followed by TABLE) (Dehaene et al., 2001). Such case-invariant priming in the VWFA was recently replicated with words made only of visually dissimilar letters that are related only by arbitrary cultural convention (e.g. a and A, g and G) (Dehaene et al., in press). This indicates that processing in the VWFA goes beyond generic processes of size and location normalization, and is capable of establishing the arbitrary links between upper- and lower-case letters.

Finally, the functional visual units that are represented in this region beyond the level of individual letters are still a matter of research (Pelli et al., 2003). Are letters combined into larger constituents specific to a given writing system, such as word-level units, syllables, or familiar clusters of letters? In a recent word priming study, we found indications that the mid-fusiform cortex can be decomposed into smaller subareas (Dehaene et al., in press). The posterior subpart, observed bilaterally, was not invariant for location: it showed priming when single letters were repeated at the same retinal location, irrespective of whether the prime word was identical to the target or was an anagram of it. Thus, this region likely holds a location-specific sublexical code. More anteriorily, priming became location invariant, though present for both words and their anagrams, suggesting a location-invariant sublexical code. Finally, even more anteriorily in the middle fusiform gyrus, there was a trend towards greater priming when the same word was presented twice compared to when it was preceded by an anagram, suggesting the presence of the units larger than the letter level. Experiments such as this may ultimately test the hypothesis that neurons in the fusiform region are tuned to progressively larger and more invariant units of words, from visual features in extrastriate cortex to broader units such as graphemes, syllables, morphemes, or even entire words as one moves anteriorily in the fusiform gyrus.

\footnotetext{
3 This holds quite generally, at least in passive viewing conditions or with tasks that require equal attention to words and to consonant strings; no difference, or even a reversal, can be observed if the task is more difficult to perform with consonant strings than with words (Cohen et al., 2003).
}

\section{Evidence from neuropsychology}

We now consider how the evidence from brain-lesioned patients supplements neuroimaging data. It may be useful to first summarize why pure alexia is relevant to the present debate. Pure alexia, also known as alexia-without-agraphia, word blindness, or agnosic alexia, is characterized by the breakdown of reading following left occipitotemporal lesions in literate adults. Such patients typically show intact production and comprehension of oral language, and they can write normally either spontaneously or to dictation. However, they show various degrees of impairment of word reading. In the most severe cases, known as global alexia, they cannot even identify single letters (Dejerine, 1892). More frequently, pure alexic patients show relatively preserved, albeit slow, letter identification abilities and resort to letter-by-letter reading strategies (for references, see Montant and Behrmann, 2000). This clinical pattern thus reflects (1) the loss of fast and parallel letter perception mechanisms and (2) in severe cases, the loss of single letter identification abilities.

The simplest account of pure alexia is that it reflects a breakdown of the visual word form system (Cohen et al., 2000; Warrington and Shallice, 1980). Characterizing the deficit and relating it to lesions of the VWFA is thus highly relevant. The VWFA hypothesis predicts that patients with pure alexia should always have lesions critically affecting the VWFA or its connections (reproducible localization). Furthermore, if the lesions are sufficiently restricted, visual word recognition should be disproportionately impaired relative to other types of visual stimuli (partial regional selectivity). Finally, the features of the deficits should indicate a loss of knowledge specific to the patient's writing system (functional specialization).

\section{Pure alexia and reproducible localization}

According to $\mathrm{P} \& \mathrm{D}$, pure alexic patients "usually have extensive left occipital lesions including the cuneus, calcarine sulcus, and lingual gyrus", and therefore "it is impossible to localize their word reading deficit to a particular area of damaged cortex". Several arguments can be raised against this pessimistic view. First, not all patients have extensive lesions blurring anatomical correlations. To take but one example, Beversdorf et al. (1997) report a case with a limited surgical excision and demonstrative postmortem data. The lesion affected the expected region of the left mid-fusiform cortex, with an extension to the lateral edge of the lingual gyrus. Second, even in patients with large lesions, the combination of lesions from multiple patients may allow to identify the necessary and sufficient regions that are reproducibly associated with pure alexia. Such work was done by Binder and Mohr (1992), Damasio and Damasio (1983), and more recently by our group (Cohen et al., 2003), with a good agreement of these studies on the lateral edge of the left fusiform gyrus. For instance, Binder and Mohr (1992) delineated a critical region by comparing the lesions of alexic patients with those of patients with a left posterior cerebral artery infarct and no reading impairment, including patients with lesions to the lingual gyrus or the cuneus. The critical region fell within the left middle fusiform gyrus. Although it appears slightly more anterior than the VWFA, methodological differences in the reconstruction of lesions may be sufficient to account for this discrepancy. In our recent work, we also found a similar region of lesion overlap in the fusiform gyrus. Although this region was admittedly larger than the VWFA strictly defined, it 
precisely encompassed the region of activation to words identified in fMRI studies of reading (Cohen et al., 2003). Thus, although the precision of the correlations should still be improved, there is good convergence towards the site of the VWFA as the anatomical correlate of pure alexia.

A factor that may blur anatomo-functional correlations is the role of white matter lesions. Our hypothesis implies that pure alexia should result either from a disruption of the VWFA itself, or from impaired projections to or from this system. The clearest example of such a deafferentation is the alexia restricted to the left half of the visual field which results from lesions of posterior callosal pathways (Suzuki et al., 1998). In such cases, we observed reduced activations of the VWFA for words presented in the left hemifield (Cohen et al., 2000; Molko et al., 2002). Similarly, a few cases of alexia restricted to the RVF have been reported, and may be due to left intrahemispheric lesions deafferenting the VWFA but sparing low-level visual regions (Castro-Caldas and Salgado, 1984). Finally, the anatomical case reported by Greenblatt (1973) may be an instance of complete deafferentation of an intact fusiform cortex (see also Cohen et al., submitted for publication). Conversely, output projections from the VWFA to lateral language areas may be interrupted during their course in the parietal white matter, providing a plausible account for the so-called subangular alexia (Greenblatt, 1976; Iragui and Kritchevsky, 1991; Pirozzolo et al., 1981). Altogether, simple considerations on the input and output pathways of the VWFA provide a detailed and consistent explanation for the variety of visual alexias seen in adult neuropsychological cases (Cohen et al., 2003).

P\&D emphasize that the two alexic patients studied by Warrington and Shallice (1980) had no lesion to the left mid-fusiform gyrus, which would argue against the role of this area in pure alexia. Actually, the extent of the patients' lesions was not reported in any detail, and the fusiform gyrus may well have been affected. One lesion was a haemorrhage large enough to cause 1 month of coma, and the other one was an invasive temporoparietal glioma which had been evolving for several years, and there is no evidence that the fusiform was spared. Even assuming that it was, these observations would not necessarily contradict the proposed model due to the possible contribution of white matter lesions. Thus, no firm anatomical argument, positive or negative, can be made from Warrington and Shallice's cases.

\section{Pure alexia and regional selectivity}

In line with their hypothesis that there is no subregion of the visual cortex specific to words, $P \& D$ further suggest that "pure alexia is the most salient manifestation of a more general visual problem". Their main argument is based on the existence of visual deficits beyond the domain of reading in patients with pure alexia.

However, such associations of deficits are known to provide only a weak source of constraints (Shallice, 1988). Given that the VWFA may contain cortical columns specialized for letter processing, mixed with columns with different preferences (Puce et al., 1999), it is likely that a disruption of the VWFA would induce some degree of impairment beyond reading (Behrmann et al., 1998a). Even assuming that there is a cortical sector highly selective for the perception of alphabetic stimuli, it would be unlikely that a spontaneous lesion should affect this region with perfect selectivity although sparing the adjacent cortex. Thus, it is hardly surprising that some degree of associated visual impair- ment can be evidenced in most alexic patients. This does not undermine our claims relative to the function of the VWFA in reading.

A second problem with P\&D's argument is that for associated deficits to have some relevance to the debate, one should not only bring those deficits to light, but also demonstrate that alexia can be reduced entirely to a single shared mechanism. However, we believe that there is presently no complete account of pure alexia in terms of a "general visual problem". The breakdown of parallel letter processing in pure alexia has been attributed to a general inability to represent multiple object parts (Farah and Wallace, 1991) or to an increased sensitivity to visual object complexity (Behrmann et al., 1998a). However, it is unclear how this may account for the frequent sparing of the recognition of complex multipart objects, faces, or digit strings in pure alexic patients. For instance, as an argument against a general impairment of parallel visual processing, Warrington and Shallice (1980) and Patterson and Kay (1982) showed that patients with letter-by-letter reading could accurately report digits flanking a letter string (e.g. 6APPLE4), even when such stimuli were flashed for $150 \mathrm{~ms}$ and comprised as many as eight intervening letters. Indeed, P\&D's hypothesis that "reading is more complex and imposes greater demands on visual processing", is not sufficiently articulate to account for double dissociations such as object agnosia without alexia (Albert et al., 1975; Gomori and Hawryluk, 1984) or prosopagnosia without alexia (e.g. McNeil and Warrington, 1993).

Furthermore, impaired identification of even single letters is common in pure alexia, ranging in severity from complete global alexia to the occurrence of occasional errors with a significant slowing down of letter naming (for a review, see Behrmann et al., 1998b; e.g. Hanley and Kay, 1996). This symptom presents a real challenge to nonspecific theories of pure alexia because it is difficult to argue that single letters are in any sense more complex than faces or drawings of objects.

\section{Pure alexia and functional specialization}

We propose that during the acquisition of literacy, the VWFA incorporates script- or language-specific arbitrary regularities. This predicts that disruption of the VWFA should result in the loss of the knowledge of regularities unique to the reading system. Several observations support this hypothesis. For instance, the patient studied by Miozzo and Caramazza (1998) was severely impaired at naming single letters, and she was unable to decide whether an upper- and lower-case letter had the same name or not. However, she could accurately discriminate real letters from visually equivalent pseudo-letters, as well as normally oriented letters from mirror-reversed letters. This suggests a loss of abstract orthographic knowledge, with preservation of a basic familiarity with individual letter shapes.

Chanoine et al. (1998) reported closely similar findings in another patient. They also observed that whenever the distractors were digits or simple geometrical shapes, the patient could easily match lower- and upper-case letters, or pick out a printed letter named by the examiner, although these tasks were severely impaired when the distractors were other letters. This dissociation between letters and digits in pure alexia dates back to Dejerine's observation, as he noted that "the patient recognizes very well all digits", although "he cannot recognize a single letter" (Cohen and Dehaene, 1995; Dejerine, 1892; Holender and Peereman, 1987; for imaging data, see also Pinel et al., 2001; Polk et al., 2002). 
Such features of pure alexia cannot be plausibly accounted for from nonspecific perceptual impairments. Rather, they reflect the breakdown of the functional specialization incorporated during the acquisition of literacy, including knowledge of the finite set of characters, the links between upper- and lower-case letters, and the organization in arbitrary subsets of symbols such as letters and digits.

\section{Conclusion}

Psychological, neuropsychological, and neuroimaging data converge to suggest that the human brain of literate subjects contains specialized mechanisms for visual word recognition (functional specialization), which map in a systematic way onto the properties of a cortical subregion of the left posterior occipitotemporal sulcus (reproducible localization). We claim that this constitutes sufficient evidence to label this region the visual word form area, even if at present the evidence for the third type of cortical specialization (regional selectivity) is not definitive. The region that activates to visual words is well delimited and occupies a fixed location relative to other regions that preferentially respond to faces, houses, or objects (Hasson et al., 2002, 2003; Puce et al., 1996). Nevertheless, most cortical patches that respond to words also activate to other stimuli such as pictures or drawings. Thus, the evidence to date favors a model of partially distributed and overlapping representation of visual categories (Haxby et al., 2000, 2001), with a landscape of partial but not absolute preferences across the ventral cortical surface. One should keep in mind that this picture results from imaging methods with limited resolution (several millimeters) and may change as one becomes able to visualize or record from small cortical patches, columns, or even single neurons. Neurophysiological and optical imaging methods indicate a high degree of local cortical specialization in inferotemporal (IT) neurons (Tanaka, 1996), with columnar clustering of cells with similar selectivity. In the macaque perirhinal cortex, specific visual experience induces development of clusters of neurons with similar stimulus preferences (Erickson et al., 2000). IT neurons are able to learn new arbitrary shapes (Logothetis et al., 1995), and arbitrary pairings of stimuli (Sakai and Miyashita, 1991), which might be an analogue of learning letters and of linking arbitrarily related upper- and lowercase letters. Likewise, the binding of letters into words might be related to the representation of complex objects in IT cortex through the coactivation of neurons tuned to their elementary parts (Tsunoda et al., 2001). Binding of letters may also be based on more holistic coding, as suggested by the recent demonstration that single IT neurons develop selectivity for learned complex shapes, above and beyond what could be expected from the additive influence of their component parts (Baker et al., 2002). Such neurophysiological mechanisms may provide a neuronal basis for the acquisition of invariant visual word recognition in humans.

Such observations predict the existence of highly specialized but patchy and distributed neuronal populations coding for alphabetic stimuli at the single-neuron level. The intermingling of such neurons with others coding for objects or faces would translate into a partial regional selectivity at the single-voxel level, which is all that we can presently measure with PET or fMRI.

One puzzling issue remains: why is there a reproducible cortical site responsive to visual words? Reading is a recent cultural activity of the human species. The 5400 years that have elapsed since its invention are too short to permit the evolution of dedicated biolog- ical mechanisms for learning to read. Visual word recognition therefore necessarily makes use of preexisting primate circuitry. We tentatively suggest that the reproducible localization of the VWFA indicates that only a small subregion of visual cortex has the optimal initial characteristics that permit it to be preempted or "recycled" for reading (Dehaene, 2004). The patch of cortex that responds to visual words is endowed with general functional properties. Those include its bias for foveal vs. peripheral images (Hasson et al., 2002), its location at an intermediate level of the hierarchy of visual areas (Grill-Spector et al., 1998) appropriate for object-level processing (Tarkiainen et al., 2002), and its sensitivity to local features vs. configural information (Gauthier et al., 2000; Lerner et al., 2001).

As to the left lateralization of the VWFA, it may result from interhemispheric differences in the processing of spatial frequencies (Kitterle and Selig, 1991) or in the invariant vs. token-specific coding of visual objects (Burgund and Marsolek, 1997). The lateralization may also imply preferential connections to lefthemispheric language areas. The conjunction of these factors may collectively conspire to define a cortical region optimally apt at hosting the functional processes of visual word recognition. Finally, it should be remembered that it takes years for children to develop expert word recognition (Aghababian and Nazir, 2000), and that the emergence of this expertise correlates with the amount of activation of the VWFA by letter strings in normal and dyslexic children (McCandliss et al., 2003; Shaywitz et al., 2002; Temple, 2002). There is thus no ready-made "module" for visual word recognition, but a progressive specialization process that capitalizes on the plasticity of the human ventral inferotemporal cortex to build the VWFA.

\section{References}

Aghababian, V., Nazir, T.A., 2000. Developing normal reading skills: aspects of the visual processes underlying word recognition. J. Exp. Child Psychol. 76, 123-150.

Albert, M.L., Reches, A., Silverberg, R., 1975. Associative visual agnosia without alexia. Neurology 25, 322-326.

Allison, T., McCarthy, G., Nobre, A., Puce, A., Belger, A., 1994. Human extrastriate visual cortex and the perception of faces words numbers and colors. Cereb. Cortex 5, 544-554.

Allison, T., Puce, A., Spencer, D.D., McCarthy, G., 1999. Electrophysiological studies of human face perception. I: Potentials generated in occipitotemporal cortex by face and non-face stimuli. Cereb. Cortex 9, 415-430.

Baker, C.I., Behrmann, M., Olson, C.R., 2002. Impact of learning on representation of parts and wholes in monkey inferotemporal cortex. Nat. Neurosci. 5, 1210-1216.

Beauregard, M., Chertkow, H., Bub, D., Murtha, S., Dixon, R., Evans, A., 1997. The neural substrate for concrete, abstract, and emotional word lexica: a positron emission tomography study. J. Cogn. Neurosci. 9, $441-461$

Behrmann, M., Nelson, J., Sekuler, E.B., 1998a. Visual complexity in letter-by-letter reading: "pure" alexia is not pure. Neuropsychologia 36, 1115-1132.

Behrmann, M., Plaut, D.C., Nelson, J., 1998b. A literature review and new data supporting an interactive account of letter-by-letter reading. Cogn. Neuropsychol. 15, 7-51.

Besner, D., 1989. On the role of outline shape and word-specific visual pattern in the identification of function words: NONE. Q. J. Exp. Psychol. 41A, 91-105.

Besner, D., Coltheart, M., Davelaar, E., 1984. Basic processes in read- 
ing: computation of abstract letter identities. Can. J. Psychol. 38, $126-134$.

Beversdorf, D.Q., Ratcliffe, N.R., Rhodes, C.H., Reeves, A.G., 1997. Pure alexia: clinical-pathologic evidence for a lateralized visual language association cortex. Clin. Neuropathol. 16, 328-331.

Binder, J.R., Mohr, J.P., 1992. The topography of callosal reading pathways. A case-control analysis. Brain 115, 1807-1826.

Booth, J.R., Burman, D.D., Meyer, J.R., Gitelman, D.R., Parrish, T.B., Mesulam, M.M., 2002a. Functional anatomy of intra- and cross-modal lexical tasks. NeuroImage 16, 7-22.

Booth, J.R., Burman, D.D., Meyer, J.R., Gitelman, D.R., Parrish, T.B., Mesulam, M.M., 2002b. Modality independence of word comprehension. Hum. Brain Mapp. 16, 251-261.

Booth, J.R., Burman, D.D., Meyer, J.R., Gitelman, D.R., Parrish, T.B., Mesulam, M.M., 2003. Relation between brain activation and lexical performance. Hum. Brain Mapp. 19, 155-169.

Bowers, J.S., Vigliocco, G., Haan, R., 1998. Orthographic, phonological, and articulatory contributions to masked letter and word priming. J. Exp. Psychol. Hum. Percept. Perform. 24, 1705-1719.

Brunswick, N., McCrory, E., Price, C.J., Frith, C.D., Frith, U., 1999. Explicit and implicit processing of words and pseudowords by adult developmental dyslexics: a search for Wernicke's Wortschatz? Brain 122, 1901-1917.

Büchel, C., Price, C., Friston, K., 1998. A multimodal language region in the ventral visual pathway. Nature 394, 274-277.

Burgund, E.D., Marsolek, C.J., 1997. Letter-case-specific priming in the right cerebral hemisphere with a form-specific perceptual identification task. Brain Cogn. 35, 239-258.

Burton, M.W., Small, S.L., Blumstein, S.E., 2000. The role of segmentation in phonological processing: an fMRI investigation. J. Cogn. Neurosci. $12,679-690$.

Castro-Caldas, A., Salgado, V., 1984. Right hemifield alexia without hemianopia. Arch. Neurol. 41, 84-87.

Castro-Caldas, A., Petersson, K.M., Reis, A., Stone-Elander, S., Ingvar, M., 1998. The illiterate brain. Learning to read and write during childhood influences the functional organization of the adult brain. Brain 121 (Pt. 6), $1053-1063$.

Chanoine, V., Teixeira Ferreira, C., Demonet, J.F., Nespoulous, J.L., Poncet, M., 1998. Optic aphasia with pure alexia: a mild form of visual associative agnosia? A case study. Cortex 34, 437-448.

Cohen, L., Dehaene, S., 1995. Number processing in pure alexia: the effect of hemispheric asymmetries and task demands. Neurocase 1, $121-137$.

Cohen, L., Dehaene, S., Naccache, L., Lehéricy, S., Dehaene-Lambertz, G., Hénaff, M.A., et al., 2000. The visual word form area: spatial and temporal characterization of an initial stage of reading in normal subjects and posterior split-brain patients. Brain 123, 291-307.

Cohen, L., Lehericy, S., Chochon, F., Lemer, C., Rivaud, S., Dehaene, S., 2002. Language-specific tuning of visual cortex? Functional properties of the Visual Word Form Area. Brain 125, 1054-1069.

Cohen, L., Martinaud, O., Lemer, C., Lehericy, S., Samson, Y., Obadia, M., et al., 2003. Visual word recognition in the left and right hemispheres: anatomical and functional correlates of peripheral alexias. Cereb. Cortex $13,1313-1333$.

Cohen, L., Henry, C., Dehaene, S., Molko, N., Lehéricy, S., Martinaud, O., et al., submitted for publication. The pathophysiology of letter-by-letter reading.

Damasio, A.R., Damasio, H., 1983. The anatomic basis of pure alexia. Neurology 33, 1573-1583.

Dehaene, S., 2004. Pre-emption of human cortical circuits by numbers and language: the "neuronal recycling" hypothesis. In: Dehaene, S., Duhamel, J.R., Hauser, M., Rizzolatti, G. (Eds.), From Monkey Brain to Human Brain. MIT Press, Cambridge, Massachusetts. Vol in press.

Dehaene, S., Naccache, L., Cohen, L., Bihan, D.L., Mangin, J.F., Poline, J.B., et al., 2001. Cerebral mechanisms of word masking and unconscious repetition priming. Nat. Neurosci. 4, 752-758.

Dehaene, S., Le Clec'H, G., Poline, J.B., Le Bihan, D., Cohen, L., 2002.
The visual word form area: a prelexical representation of visual words in the fusiform gyrus. NeuroReport 13, 321-325.

Dehaene, S., Jobert, A., Naccache, L., Ciuciu, P., Poline, J.B., Le Bihan, D., et al., in press. Letter binding and invariant recognition of masked words: behavioral and neuroimaging evidence. Psychol. Sci.

Dejerine, J., 1892. Contribution à l'étude anatomo-pathologique et clinique des différentes variétés de cécité verbale. Mém. Soc. Biol. 4, $61-90$.

Donnenwerth-Nolan, S., Tanenhaus, M.K., Seidenberg, M.S., 1981. Multiple code activation in word recognition: evidence from rhyme monitoring. J. Exp. Psychol. Hum. Learn. Mem. 7, 170-180.

Downing, P.E., Jiang, Y., Shuman, M., Kanwisher, N., 2001. A cortical area selective for visual processing of the human body. Science 293, $2470-2473$.

Erickson, C.A., Bharathi, J., Desimone, R., 2000. Clustering of perirhinal neurons with similar properties following visual experience in adult monkeys. Nat. Neurosci. 3, 1143-1148.

Farah, M.J., Wallace, M.A., 1991. Pure alexia as a visual impairment: a reconsideration. Cogn. Neuropsychol. 8, 313-334.

Fiez, J.A., Balota, D.A., Raichle, M.E., Petersen, S.E., 1999. Effects of lexicality, frequency, and spelling-to-sound consistency on the functional anatomy of reading. Neuron 24, 205-218.

Gauthier, I., Tarr, M.J., Moylan, J., Skudlarski, P., Gore, J.C., Anderson, A.W., 2000. The fusiform "face area" is part of a network that processes faces at the individual level. J. Cogn. Neurosci. 12, 495-504.

Giraud, A.L., Price, C.J., 2001. The constraints functional neuroanatomy places on classical models of auditory word processing. J. Cogn. Neurosci. $13,754-765$.

Gomori, A.J., Hawryluk, G.A., 1984. Visual agnosia without alexia. Neurology 34, 947-950.

Greenblatt, S.H., 1973. Alexia without agraphia or hemianopsia. Anatomical analysis of an autopsied case. Brain 96, 307-316.

Greenblatt, S.H., 1976. Subangular alexia without agraphia or hemianopsia. Brain Lang. 3, 229-245.

Grill-Spector, K., Malach, R., 2001. fMR-adaptation: a tool for studying the functional properties of human cortical neurons. Acta Psychol. (Amst.) 107, 293-321.

Grill-Spector, K., Kushnir, T., Hendler, T., Edelman, S., Itzchak, Y., Malach, R., 1998. A sequence of object-processing stages revealed by fMRI in the human occipital lobe. Hum. Brain Mapp. 6, 316-328.

Hallé, P.A., Chéreau, C., Segui, J., 2000. Where is the/b/in "absurde" [apsyrd]? It is in French listeners' minds. J. Mem. Lang. 43, 618-639.

Hanley, J.R., Kay, J., 1996. Reading speed in pure alexia. Neuropsychologia 34, 1165-1174.

Hasson, U., Levy, I., Behrmann, M., Hendler, T., Malach, R., 2002. Eccentricity bias as an organizing principle for human high-order object areas. Neuron 34, 479-490.

Hasson, U., Harel, M., Levy, I., Malach, R., 2003. Large-scale mirrorsymmetry organization of human occipito-temporal object areas. Neuron 37, 1027-1041.

Haxby, J.V., Ishai, I.I., Chao, L.L., Ungerleider, L.G., Martin, I.I., 2000. Object-form topology in the ventral temporal lobe. Response to I. Gauthier (2000). Trends Cogn. Sci. 4, 3-4.

Haxby, J.V., Gobbini, M.I., Furey, M.L., Ishai, A., Schouten, J.L., Pietrini, P., 2001. Distributed and overlapping representations of faces and objects in ventral temporal cortex. Science 293, 2425-2430.

Holender, D., Peereman, R., 1987. Differential processing of phonographic and logographic single-digit numbers by the two hemispheres. In: Deloche, G., Seron, X. (Eds.), Mathematical Disabilities: A Cognitive Neuropsychological Perspective. Lawrence Erlbaum Associates, Hillsdale, NJ, pp. 43-86.

Iragui, V.J., Kritchevsky, M., 1991. Alexia without agraphia or hemianopia in parietal infarction. J. Neurol., Neurosurg. Psychiaxtry 54, 841-842.

Kanwisher, N., McDermott, J., Chun, M.M., 1997. The fusiform face area: a module in human extrastriate cortex specialized for face perception. J. Neurosci. 17, 4302-4311. 
Kitterle, F.L., Selig, L.M., 1991. Visual field effects in the discrimination of sine-wave gratings. Percept. Psychophys. 50, 15-18.

Kreiman, G., Koch, C., Fried, I., 2000. Category-specific visual responses of single neurons in the human medial temporal lobe. Nat. Neurosci. 3, 946-953.

Lavidor, M., Ellis, A.W., 2002. Word length and orthographic neighborhood size effects in the left and right cerebral hemispheres. Brain Lang. $80,45-62$.

Lavidor, M., Babkoff, H., Faust, M., 2001. Analysis of standard and nonstandard visual word format in the two hemispheres. Neuropsychologia $39,430-439$.

Lerner, Y., Hendler, T., Ben-Bashat, D., Harel, M., Malach, R., 2001. A hierarchical axis of object processing stages in the human visual cortex. Cereb. Cortex 11, 287-297.

Logothetis, N.K., Pauls, J., Poggio, T., 1995. Shape representation in the inferior temporal cortex of monkeys. Curr. Biol. 5, 552-563.

Marinkovic, K., Dhond, R.P., Dale, A.M., Glessner, M., Carr, V., Halgren, E., 2003. Spatiotemporal dynamics of modality-specific and supramodal word processing. Neuron 38, 487-497.

Mayall, K., Humphreys, G.W., Olson, A., 1997. Disruption to word or letter processing? The origins of case-mixing effects. J. Exper. Psychol., Learn., Mem., Cogn. 23, 1275-1286.

McCandliss, B.D., Cohen, L., Dehaene, S., 2003. The Visual Word Form Area: expertise for reading in the fusiform gyrus. Trends Cogn. Sci. 7, 293-299.

McNeil, J.E., Warrington, E.K., 1993. Prosopagnosia: a face-specific disorder. Q. J. Exp. Psychol., A 46, 1-10.

Miozzo, M., Caramazza, A., 1998. Varieties of pure alexia: the case of failure to access graphemic representations. Cogn. Neuropsychol. 15, $203-238$.

Molko, N., Cohen, L., Mangin, J.F., Chochon, F., Lehéricy, S., Le Bihan, D., et al., 2002. Visualizing the neural bases of a disconnection syndrome with diffusion tensor imaging. J. Cogn. Neurosci. 14, $629-636$

Montant, M., Behrmann, M., 2000. Pure alexia. Neurocase 6, 265-294.

Naccache, L., Dehaene, S., 2001. The priming method: imaging unconscious repetition priming reveals an abstract representation of number in the parietal lobes. Cereb. Cortex 11, 966-974.

Nazir, T.A., 2000. Traces of print along the visual pathway. In: Kennedy, A., Radach, R., Heller, D., Pynte, J. (Eds.), Reading as a Perceptual Process. Elsevier, Amsterdam, pp. 3-22.

Nobre, A.C., Allison, T., McCarthy, G., 1994. Word recognition in the human inferior temporal lobe. Nature 372, 260-263.

Nobre, A.C., Allison, T., McCarthy, G., 1998. Modulation of human extrastriate visual processing by selective attention to colours and words. Brain 121, 1357-1368.

Paap, K.R., Newsome, S.L., Noel, R.W., 1984. Word shape's in poor shape for the race to the lexicon. J. Exp. Psychol. Hum. Percept. Perform. 10, 413-428.

Patterson, K., Kay, J., 1982. Letter-by-letter reading: psychological descriptions of a neurological syndrome. Q. J. Exp. Psychol. 34A, 411-441.

Paulesu, E., McCrory, E., Fazio, F., Menoncello, L., Brunswick, N., Cappa, S.F., et al., 2000. A cultural effect on brain function. Nat. Neurosci. 3, $91-96$.

Pelli, D.G., Farell, B., Moore, D.C., 2003. The remarkable inefficiency of word recognition. Nature 423, 752-756.

Petersen, S.E., Fiez, J.A., 1993. The processing of singlewords studied with positron emission tomography. Annu. Rev. Neurosci. 16, 509-530.

Petersen, S.E., Fox, P.T., Snyder, A.Z., Raichle, M.E., 1990. Activation of extrastriate and frontal cortical areas by visual words and word-like stimuli. Science 249, 1041-1044.

Pinel, P., Dehaene, S., Riviere, D., LeBihan, D., 2001. Modulation of parietal activation by semantic distance in a number comparison task. NeuroImage 14, 1013-1026.

Pirozzolo, F.J., Kerr, K.L., Obrzut, J.E., Morley, G.K., Haxby, J.V., Lundgren, S., 1981. Neurolinguistic analysis of the language abilities of a patient with a gdouble disconnection syndromeh: a case of subangular alexia in the presence of mixed transcortical aphasia. J. Neurol., Neurosurg. Psychiatry 44, 152-155.

Polk, T.A., Farah, M.J., 2002. Functional MRI evidence for an abstract, not perceptual, word-form area. J. Exp. Psychol. Gen. 131, 65-72.

Polk, T.A., Stallcup, M., Aguirre, G.K., Alsop, D.C., D’Esposito, M., Detre, J.A., et al., 2002. Neural specialization for letter recognition. J. Cogn. Neurosci. 14, 145-159.

Price, C.J., Devlin, J.T., 2003. The myth of the visual word form area. NeuroImage 19, 473-481.

Price, C.J., Wise, R.J.S., Frackowiak, R.S.J., 1996. Demonstrating the implicit processing of visually presented words and pseudowords. Cereb. Cortex 6, 62-70.

Price, C.J., Winterburn, D., Giraud, A.L., Moore, C.J., Noppeney, U., 2003. Cortical localisation of the visual and auditory word form areas: a reconsideration of the evidence. Brain Lang. 86, 272-286.

Puce, A., Allison, T., Asgari, M., Gore, J.C., McCarthy, G., 1996. Differential sensitivity of human visual cortex to faces, letterstrings, and textures: a functional magnetic resonance imaging study. J. Neurosci. $16,5205-5215$.

Puce, A., Allison, T., McCarthy, G., 1999. Electrophysiological studies of human face perception. III: Effects of top-down processing on facespecific potentials. Cereb. Cortex 9, 445-458.

Rees, G., Russell, C., Frith, C.D., Driver, J., 1999. Inattentional blindness versus inattentional amnesia for fixated but ignored words. Science 286 , 2504-2507.

Reicher, G.M., 1969. Perceptual recognition as a function of meaningfulness of stimulus material. J. Exp. Psychol. 81, 274-280.

Riesenhuber, M., Poggio, T., 1999. Hierarchical models of object recognition in cortex. Nat. Neurosci. 2, 1019-1025.

Rumelhart, D.E., McClelland, J.L., 1982. An interactive activation model of context effects in letter perception: Part 2. The contextual enhancement effect and some tests and extensions of the model. Psychol. Rev. $89,60-94$.

Sakai, K., Miyashita, Y., 1991. Neural organization for the long-term memory of paired associates. Nature 354, 152-155.

Salmelin, R., Service, E., Kiesilä, P., Uutela, K., Salonen, O., 1996. Impaired visual word processing in dyslexia revealed with magnetoencephalography. Ann. Neurol. 40, 157-162.

Shallice, T., 1988. From Neuropsychology to Mental Structure. Cambridge Univ. Press, Cambridge.

Shaywitz, B.A., Shaywitz, S.E., Pugh, K.R., Mencl, W.E., Fulbright, R.K., Skudlarski, P., et al., 2002. Disruption of posterior brain systems for reading in children with developmental dyslexia. Biol. Psychiatry 52, $101-110$

Simos, P.G., Breier, J.I., Fletcher, J.M., Foorman, B.R., Castillo, E.M., Papanicolaou, A.C., 2002. Brain mechanisms for reading words and pseudowords: an integrated approach. Cereb. Cortex 12, 297-305.

Sowell, E.R., Thompson, P.M., Rex, D., Kornsand, D., Tessner, K.D., Jernigan, T.L., et al., 2002. Mapping sulcal pattern asymmetry and local cortical surface gray matter distribution in vivo: maturation in perisylvian cortices. Cereb. Cortex 12, 17-26.

Spoehr, K.T., Smith, E.E., 1975. The role of orthographic and phonotactic rules in perceiving letter patterns. J. Exp. Psychol. Hum. Percept. Perform. 104, 21-34.

Suzuki, K., Yamadori, A., Endo, K., Fujii, T., Ezura, M., Takahashi, A., 1998. Dissociation of letter and picture naming resulting from callosal disconnection. Neurology 51, 1390-1394.

Taft, M., Hambly, G., 1985. The influence of orthography on phonological representations in the lexicon. J. Mem. Lang. 24, 320-335.

Tanaka, K., 1996. Inferotemporal cortex and object vision. Annu. Rev. Neurosci. 19, 109-139.

Tarkiainen, A., Helenius, P., Hansen, P.C., Cornelissen, P.L., Salmelin, R., 1999. Dynamics of letter string perception in the human occipitotemporal cortex. Brain 122, 2119-2132.

Tarkiainen, A., Cornelissen, P.L., Salmelin, R., 2002. Dynamics of visual feature analysis and object-level processing in face versus letter-string perception. Brain 125, 1125-1136. 
Temple, E., 2002. Brain mechanisms in normal and dyslexic readers. Curr. Opin. Neurobiol. 12, 178-183.

Thompson, P.M., Schwartz, C., Lin, R.T., Khan, A.A., Toga, A.W., 1996. Three-dimensional statistical analysis of sulcal variability in the human brain. J. Neurosci. 16, 4261-4274.

Tsunoda, K., Yamane, Y., Nishizaki, M., Tanifuji, M., 2001. Complex objects are represented in macaque inferotemporal cortex by the combination of feature columns. Nat. Neurosci. 4, 832-838.

Wagner, A.D., Schacter, D.L., Rotte, M., Koutstaal, W., Maril, A., Dale, A.M., et al., 1998. Building memories: remembering and forgetting of verbal experiences as predicted by brain activity. Science 281, $1188-1191$.

Wang, G., Tanaka, K., Tanifuji, M., 1996. Optical imaging of functional organization in the monkey inferotemporal cortex. Science 272, $1665-1668$.

Warrington, E.K., Shallice, T., 1980. Word-form dyslexia. Brain 103, $99-112$.

Weekes, B.S., 1997. Differential effects of number of letters on word and nonword naming latency. Q. J. Exp. Psychol. 50A, 439-456.

Xu, B., Grafman, J., Gaillard, W.D., Ishii, K., Vega-Bermudez, F., Pietrini, P., et al., 2001. Conjoint and extended neural networks for the computation of speech codes: the neural basis of selective impairment in reading words and pseudowords. Cereb. Cortex 11, 267-277.

Ziegler, J.C., Ferrand, L., 1998. Orthography shapes the perception of speech: the consistency effect in auditory word recognition. Psychon. Bull. Rev. 5, 683-689. 